\title{
Optimización de máquinas reconfigurables CNC por medio de interfaz de control inteligente
}

Manuel Sanabria ${ }^{7}$

Sanabria, M. Optimización de máquinas reconfigurables CNC por medio de interfaz de control inteligente. Tecnología en Marcha. Vol. 33, especial Movilidad estudiantil. Pág 141-154.

doi) https://doi.org/10.18845/tm.v33i7.5489 


\section{Palabras clave}

FPGA; PID; Micro-Máquinas Herramienta Reconfigurable; Eficiencia; Tiempo Real; Actuador eléctrico.

\section{Resumen}

En este documento será expresado el funcionamiento de las maquinas reconfigurables CNC, su implementación, además de un desarrollo de control inteligente desarrollado en el Tecnológico de Estudios Superiores de Monterrey, a lo largo del segundo semestre del 2015. Este documento es en sí mismo un ejemplo de los diferentes controles que se pueden implementar en las maquinasCNC.

Para realizar una ejemplificación adecuada se adjuntarán gráficos e imágenes que permitan detallar el control y los beneficios de su implementación con el fin de optimizar la operación de la maquinasCNC.

\section{Abstract}

This document expressed the operation of the CNC reconfigurable machines, their implementation, in addition to a development of intelligent control developed in the Tecnológico de Estudios Superiores de Monterrey, during second semester of 2015. This document is an example of the controllers that can be implemented in CNCmachines.

To perform an adequate exemplification to adjust the images and images that adjust to the control requirements and the benefits of their implementation to optimize the operation of the CNC machines

\section{Introducción}

Durante los últimos años, centros de investigación e instituciones de diferentes países han realizado investigaciones sobre el diseño y desarrollo de máquinas-herramienta CNC reconfigurables; términos como "máquinas-herramienta de escritorio" y "micro máquinas herramienta híbridas" han surgido como respuesta a estos esfuerzos, logrando resultados en reducción del tamaño de la máquina- herramienta, ahorro de energía, espacio de trabajo, recursos materiales de la máquina-herramienta, así como ayuda para preservar el medio ambiente [1] [2]. Sin embargo, uno de los grandes retos al que se han enfrentado, es ser capaces de ofrecer sistemas de control que permitan obtener un alto grado de precisión para el maquinado de partes donde la unidad de dimensión es la micra, un factor alrededor de 25 veces más pequeño que el que puede ser logrado con el maquinado convencional.

Los actuadores eléctricos son los principales elementos de control de una máquina-herramienta CNC, ya que estos dispositivos cuya función es proporcionar la fuerza para mover un dispositivo mecánico, como lo son las herramientas de corte, la tabla, el husillo, etc. Las características de control, sencillez y precisión de los accionamientos eléctricos han hecho que sean los más implementados en las máquinas- herramienta CNC.

Dentro de los actuadores eléctricos pueden distinguirse tres tipos diferentes:

Motores de corriente directa (CD):

- Controlados porinducción.

- Controlados porexcitación. 
Motores de corriente alterna (CA):

- Síncronos.

- Asíncronos.

Motores paso a paso.

Motores lineales.

Para las máquinas-herramienta, los actuadores eléctricos son la base de control de los coordinados movimientos que se requiere para el proceso de maquinado, la corriente, la velocidad y la posición son las principales variables de control de un sistema de manufactura. Los actuadores eléctricos tienen características de desempeño particulares, y puntos de operación nominales que garantizan el máximo aprovechamiento y trabajo generado. La eficiencia es uno de los principales parámetros que se analizan en este trabajo para el control de micro-máquinas herramienta reconfigurables, y por otra parte el gasto energético, ya que estas dos variables son dependientes del desempeño y precisión de la micro-máquina herramienta reconfigurable.

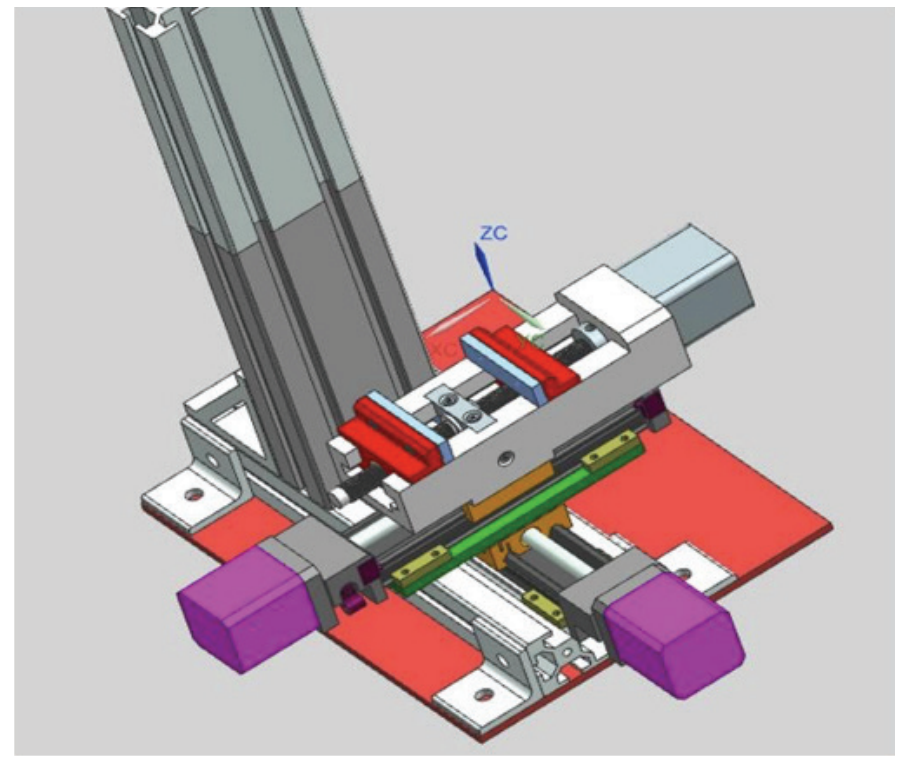

Figura 1. Máquina herramienta CNC reconfigurable, ensamble modo fresa.

En los procesos de maquinado, la exactitud del movimiento de la herramienta de corte depende del desempeño del controlador sobre los diferentes ejes de movimiento de la máquinaherramienta para minimizar los errores de posición generados sobre movimientos lineales y de contorneado. Tradicionalmente la precisión del movimiento lineal se mejora con la aplicación de lazos de realimentación y de alimentación hacia adelante. Los controladores PID (ProporcionalIntegral- Derivativo) permiten tener buena precisión y simplicidad, además de ser portables y fácilmente reconfigurables.

El interpolador elemento dentro del Control numérico por computadora, el cual hace el rol de generador de los datos de movimiento de ejes a partir del bloque de datos generados por el intérprete y es uno de los componentes claves en un CNC, este refleja su exactitud y precisión. Por lo tanto, el interpolador requiere las siguientes características para que pueda generar el desplazamiento y la velocidad con éxito para múltiples ejes de forma de la pieza y la velocidad de avance predefinido [3]. 
1. Los datos del interpolador deben estar cerca de la forma de la pieza real.

2. El interpolador debe considerar la limitación de la velocidad debido a la estructura de la máquina y las especificaciones del servo mientras calcula la velocidad [3].

Los interpoladores pueden ser implementados en hardware y software, con la evolución del CNC los interpoladores por lo general se trabajan en software debido a los algoritmos complejos que se pueden programar. Los interpoladores se clasifican en: (1) Reference pulse method y (2) Sampled-data method.

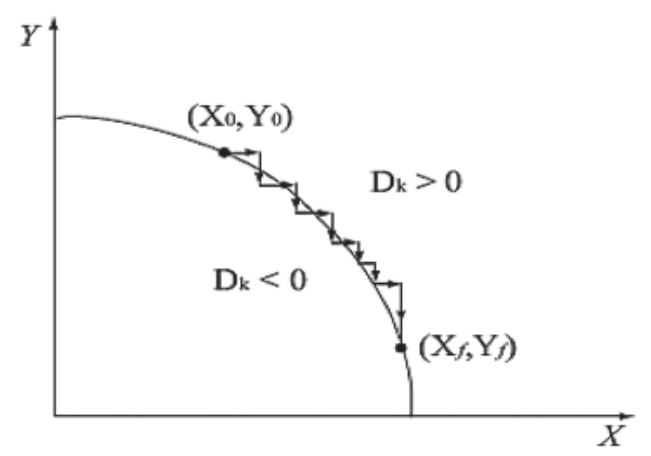

Figura 2. Comportamiento del interpolador stairs approximation.

Para un círculo, suponga que la herramienta llegue a la posición $\left(X_{k}, Y_{k}\right)$ después de ith la iteración. En este algoritmo, la variable $D_{k}$ se calcula por la ecuación:

$$
\mathrm{D}_{\mathrm{k}}=\mathrm{X}_{\mathrm{k}}^{2}+\mathrm{Y}_{\mathrm{k}}^{2}-\mathrm{R}^{2}
$$

La dirección de un paso se determina sobre la base de $D_{k}$, la dirección circular mandado, y el cuadrante donde el movimiento se realiza. Por ejemplo, si el movimiento circular se lleva a cabo en sentido horario en el primer cuadrante, el algoritmo ejecutado se explica:

1. $D_{k}<0$ : Este caso significa que la posición $\left(X_{k}, Y_{k}\right)$ se encuentra en el interior de un círculo $y$, en este caso, el paso se mueve en la dirección positiva del eje $X$.

2. $D_{k}>0$ : Este caso significa que la posición $\left(X_{k}, Y_{k}\right)$ se encuentra en el exterior de un círculo $y$, en este caso, el paso se mueve en la dirección negativa del eje $Y$

3. $D_{k}=0$ : Una de las reglas anteriores pueden ser arbitrariamente seleccionadas yaplicadas. Este algoritmo requiere una pequeña cantidad de cálculo y menos espacio de memoria.

Sin embargo, se requieren numerosas repeticiones y, el número de pasos de iteración, que es calculado con la ec. 2.

$$
\mathrm{N}=\left|\mathrm{X}_{0}-\mathrm{X}_{\mathrm{f}}\right|+\left|\mathrm{Y}_{0}-\mathrm{Y}_{\mathrm{f}}\right|,\left(\mathrm{X}_{0}, \mathrm{Y}_{0}\right)
$$

En el caso de interpolar un cuarto de circulo con radio $R$. El total de número de iteraciones, $N$ es $2 R$, con el fin de mantener la velocidad que se fija $V_{l}(\mathrm{BLU} / \mathrm{sec})$, la interpolación debe repetirse con la frecuencia conforma la ec.3. 


$$
\frac{\mathrm{f}_{0}}{\mathrm{~V}_{1}}=\frac{2 \mathrm{R}}{\pi \mathrm{R} / 2}=4 \pi
$$

El control computarizado no puede operar en tiempo continuo pues sus instrucciones se ejecutan de acuerdo a un reloj que administra sus intervalos de tiempo, de esta manera no es posible considerar un valor instantáneo de tiempo en la ejecución del control, sino del intervalo de tiempo en que ocurrió algún evento. Además, pensando en un sistema discreto completo, la adquisición de señales analógicas y su conversión a un formato digital toma cierta cantidad de tiempo, esto implica que la muestra analógica adquirida en un instante será diferente a la que se encuentra presente como entrada una vez que el controlador genere unasalida.

En el siguiente trabajo se muestra un análisis comparativo de plataformas de control PID en un dispositivo cRIO-9074 de National Instruments que incorpora una FPGA Virtex II hasta 400MHz, programado con LabVIEW FPGA. Para fines de comparación, se ha programado el mismo controlador PID en LabVIEW 2013 sobre un sistema operativo Windows 8 de 64 bits. La interfaz electrónica de entradas/salidas se ha realizado para el caso de la computadora, a través de una tarjeta de adquisición de datos NI USB-6211 las condiciones de prueba son las mismas en todos los casos.

\section{Controladores de movimiento de máquinas-herramienta}

\section{$\mathrm{CNC}$}

Existen diversas técnicas de control automático tradicionales que en la actualidad se encuentran en un estado de sinergia con técnicas modernas e inteligentes, para lograr mejores resultados de desempeño de control. Existe una gran cantidad de algoritmos de control aplicados en máquinas-herramienta CNC (Control Numérico por Computadora). En las últimas décadas la evolución de las máquinas-herramienta CNC está en función de las mejoras de los algoritmos de control, entre otros aspectos. Diferentes investigadores han enfocado sus esfuerzos y trabajos al desarrollo e implementación de nuevos controladores de movimiento en máquinas-herramienta CNC. Koren [4] realiza una evaluación de los servo-controladores y los evalúa a través de diferentes variables características del proceso de maquinado y del estado de la máquinaherramienta CNC. Clasifica los servo- controladores de la siguienteforma:

- Controladores de realimentación (feedback controllers) que pueden ser P, PID y realimentación de estados (state-feedbackcontroller).

- Controladores de alimentación hacia adelante como lo son zero phase tracking controller (ZPECT) e inverse compensation filter (IKF).

- Controlador Cross-Coupling(CCC).

- Otros (control óptimo, control predictivo, control adaptativo y controlrepetitivo).

Generalmente son 3 las estrategias de control de máquinas- herramienta CNC como lo es: (a) point-point control, (b) tracking control y (c) contour control como se muestra en la figura 2. 


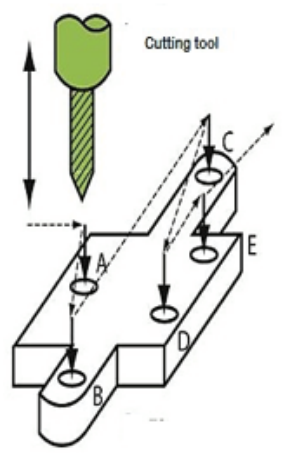

(a) Point to point control

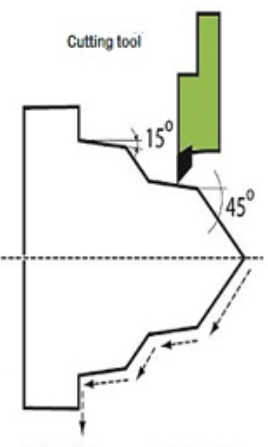

(b) Tracking control

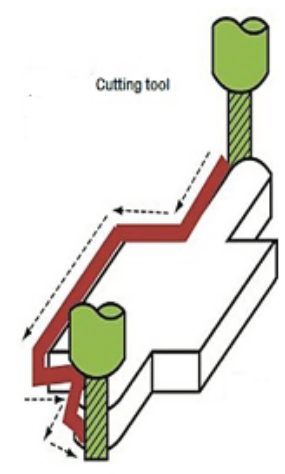

(c) Contour control

Figura 3. Estrategias de control de máquinas-herramienta CNC [5].

En una micro-máquina herramienta, cada eje de movimiento se maneja por un lazo de control independiente (figura 3). La variable a controlar representa la posición del eje que es realimentada y comparada con la referencia de entrada, que es la posición deseada. La señal resultante de la compensación del error, es la señal de accionamiento del motor por medio de un controlador, de tal manera que éste compense el error de posición que se genera. El control más simple es aquel donde la señal de salida es proporcional a la señal del error y es el llamado proporcional [4].

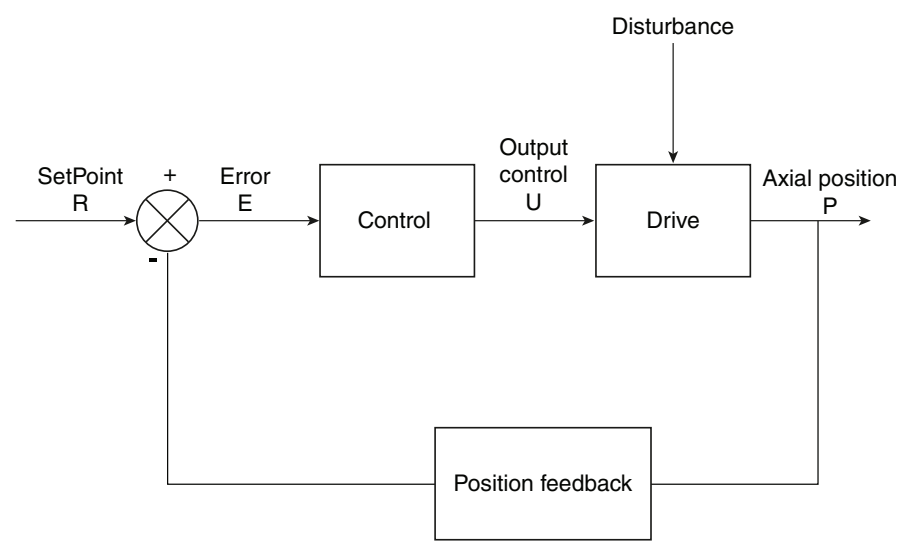

Figura 4. Diagrama de bloques de un lazo de control de posición utilizado en las micro-máquinas herramienta [6].

Por otra parte, es conveniente también garantizar una exactitud razonable y una robustez elevada contra las perturbaciones. Cada accionamiento en la micro-máquina herramienta está sujeto a errores inevitables entre la posición actual y la posición deseada, debido a la dinámica del sistema y errores debido a perturbaciones externas que ocurren durante un maquinado, dichos errores se corrigen con el controlador de posición entonces es un sistema que puede ser compensado gracias a la acción del control.

\section{Tipos de mecanizado}

A partir del desarrollo tecnológico de las máquinas-herramienta $\mathrm{CNC}$, hoy en día se distinguen diferentes tipos de procesos de mecanizado en función de diferentes variables, tales como exactitud, precisión, volumen de metal cortado por unidad de tiempo, etc. A continuación, se hará una breve descripción de los procesos de mecanizado. 
El mecanizado convencional, consiste en maquinar una determinada pieza utilizando las máquinas-herramientas convencionales, es decir, sin la utilización de controles avanzados. Esto implica la obtención de dimensiones con tolerancias entre $200 \mu \mathrm{m}$ y $50 \mu \mathrm{m}$. El mecanizado de precisión consiste en efectuar el proceso de corte de metal, para obtener tolerancias muy precisas, que se encuentran en el rango entre $5 \mu \mathrm{m}$ y $0.5 \mu \mathrm{m}$ y rugosidad superficial inferior a los $0.18 \mu \mathrm{m}$. Existe el denominado maquinado de ultra-precisión, donde se alcanzan entre $0.05 \mu \mathrm{m}$ y $0.005 \mu \mathrm{m}$ de precisión [7].

Actualmente, el maquinado de alta velocidad (MAV) tiene diversas definiciones. No significa obligatoriamente mecanizar a altas revoluciones del husillo, ya que muchas de las aplicaciones se realizan con velocidades de husillo moderadas (3000 - 6000 rpm) y herramientas de gran diámetro (25 a $30 \mathrm{~mm}$ ). Las condiciones del proceso (velocidad de corte, avance, profundidades de corte radial y axial,etc.) dependerán del material a mecanizar, así como de las máquinas y herramientas disponibles. Por tanto, para cada tipo de material existirá un rango de velocidad de rotación del husillo para el MAV [7].

\section{Micro-máquina herramienta reconfigurable}

La micro máquina-herramienta reconfigurable (mMHR), fue diseñada bajo el concepto reconfigurable, que consiste en obtener varias funciones para el mecanizado de piezas con solo reconfigurar el posicionamiento y cambiar la herramienta de corte. Las configuraciones posibles son: Taladradora, Fresadora y Torno (ver figura 5). A continuación, se enumeran los elementos que integran la micro-máquina herramienta reconfigurable (ver figura1):

1. BasePrincipal.

2. Soporte para reconfiguraciónvertical

3. BaseReconfigurable

4. Base para soporte deejes

5. Ángulo para soporte de ejey

6. Torre soporte demordaza

7. Base sujetadora d-e abrazadera dehusillo

8. Abrazadera dehusillo

9. Husillo

10. Mordaza

11. Actuador eléctricolineal

12. Portaactuador

13. Cilindro sujetador demordaza

14. Herramienta decorte

15. Actuador movimiento ejex

16. Actuador movimiento ejey

17. Actuador movimiento ejez

18. Buril

19. Inserto

20. Portaburil 


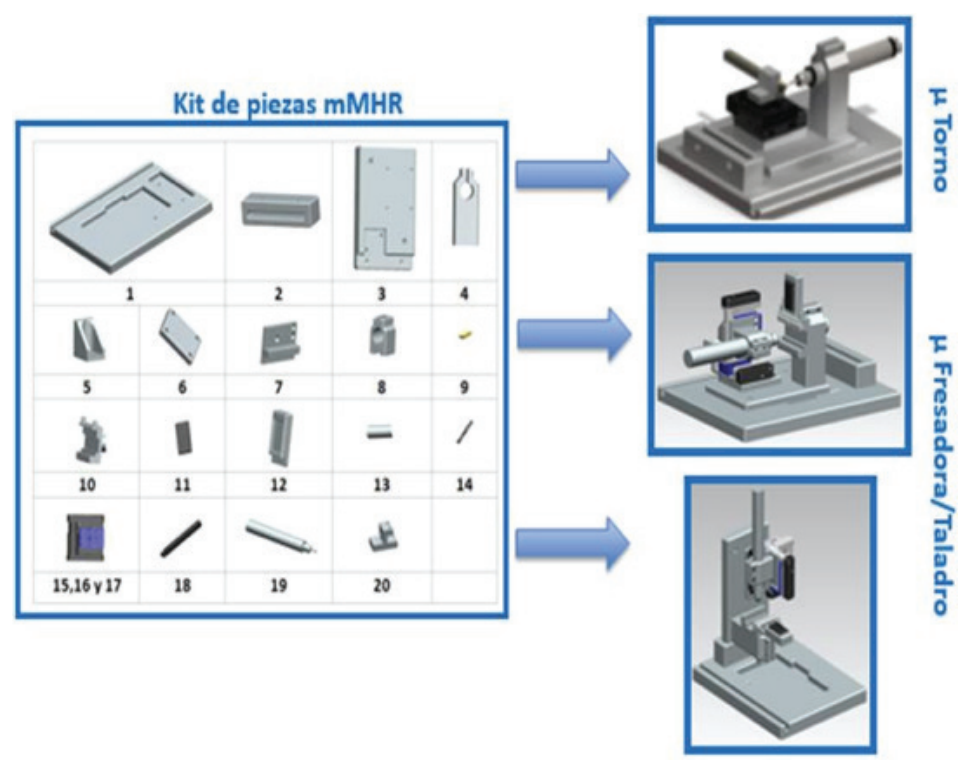

Figura 5. Elementos que integran la micro-máquina herramienta reconfigurable.

\section{Sistemas digitales para control discreto}

El control computarizado en un sistema operativo multipropósito (ejemplo: Windows 8) no puede operar en tiempo real pues sus instrucciones se ejecutan de acuerdo con un reloj que administra sus intervalos, de esta manera no es posible hablar de un valor determinístico de tiempo en la ejecución del control sino del intervalo de tiempo en que ocurrió algún evento. Además, pensando en un sistema discreto completo, la adquisición de señales analógicas y su conversión a un formato digital tomará cierta cantidad de tiempo, esto implica que la muestra analógica adquirida en un instante será diferente a la que se encuentra presente como entrada una vez que el controlador ofrezca una salida.

Para implementar un controlador en tiempo real, las FPGAs (Field Programable Field Array) resultan adecuados pues permiten el cómputo de operaciones aritméticas a altas velocidades y en paralelo, haciendo que se respeten intervalos de tiempo real reducidos [8]. Si bien es posible utilizar microcontroladores, microprocesadores, DSPs (Digital Signal Processor) o ASICs (Application-specific Integrated Circuit), la velocidad de procesamiento, el manejo de entradas/ salidas y circuitos periféricos, así como el costo reducido y la capacidad para reprogramarse entre otros, hace que las FPGAs sean una opción completa para cumplir con los propósitos garantizar en tiempo real el control [9].

\section{Control dedicado en plataformas digitales}

El control dedicado (digital) se refiere a la integración de un dispositivo con capacidad de procesamiento numérico sobre una planta, cuyas tareas están restringidas de manera exclusiva a la adquisición de datos, cómputo de valores de control y generación de salidas eléctricas hacia los actuadores. Limitar las tareas de un dispositivo y conocer el tiempo preciso que requiere cada una, hace que se cumplan los objetivos de tiempo real necesarios en la aplicación de técnicas de control discreto para tener una respuesta determinística; si el cálculo se realiza en paralelo y con altas tasas de cómputo, entonces el periodo de tiempo mínimo que el controlador puede alcanzarmejoras. 
Como se planteó anteriormente, las FPGAs cumplen con las características mencionadas anteriormente, siendo idóneas para su aplicación en un control como el que se presenta en éste artículo, sin embargo, el uso de un controlador dedicado resta versatilidad a la aplicación final que ejecuta un operador. De esta forma, pueden abordarse dos perspectivas para proveer una interfaz competitiva.

- Topología maestro-esclavo

- Uso de sistemas operativos de tiemporeal

La topología maestro esclavo es la que involucra un cliente que comúnmente corre un sistema operativo convencional donde se presenta la interfaz de usuario, un controlador dedicado y un canal de comunicación entre ellos. De esta manera, los valores de consigna se escriben desde la interfaz, son transferidos a través del canal de comunicación y el controlador ejecuta el algoritmo de control determinado. Dependiendo de la aplicación, el controlador podría reportar su estado actual o solamente fallos y alarmas (ambas perspectivas difieren evidentemente en la administración del tiempo en el controlador).

\section{Implementación}

La propuesta principal demuestra que el desempeño de un controlador digital implementado sobre una plataforma en la capa de aplicación de un sistema operativo convencional y de propósito general (Windows 8), se ve superado ampliamente por una plataforma dedicada en tiempo real y que el aseguramiento de intervalos de tiempo real cada vez más cortos favorece la precisión final del sistema, evaluado con pruebas de maquinado sobre diferentesmateriales.

La técnica de control utilizada consiste en un PID paralelo discreto programado para ambas plataformas con la misma aritmética, Para el diseño digital se considera la siguiente ecuación continua del PID:

$$
\operatorname{PID}(s)=e(s)\left(K_{p}+\frac{K_{i}}{s}+K_{d} s\right)
$$

Cuya transformación discreta se realiza con la transformada bilineal o método de Tustin, para obtener:

$$
\begin{gathered}
\mathrm{K}_{\mathrm{B}}=\frac{2 \mathrm{~K}_{\mathrm{i}} \mathrm{T}_{\mathrm{s}}^{2}-8 \mathrm{~K}_{\mathrm{d}}}{2 \mathrm{~T}_{\mathrm{s}}} \\
\mathrm{K}_{\mathrm{C}}=\frac{2 \mathrm{~K}_{\mathrm{i}} \mathrm{T}_{\mathrm{s}}^{2}-2 \mathrm{~K}_{\mathrm{p}} \mathrm{T}_{\mathrm{s}}+4 \mathrm{~K}_{\mathrm{d}}}{2 \mathrm{~T}_{\mathrm{s}}} \\
\operatorname{PID}(\mathrm{z})=\mathrm{e}(\mathrm{z})\left(\frac{\mathrm{K}_{\mathrm{A}} \mathrm{z}^{2}+\mathrm{K}_{\mathrm{B}} \mathrm{z}+\mathrm{K}_{\mathrm{c}}}{\mathrm{z}^{2}-1}\right)
\end{gathered}
$$

Empleando las ecuaciones en recurrencia se tiene:

$$
\mathrm{PID}(\mathrm{k})=\mathrm{K}_{\mathrm{A}} \mathrm{e}(\mathrm{k})+\mathrm{K}_{\mathrm{B}} \mathrm{e}(\mathrm{k}-1)+\mathrm{K}_{\mathrm{C}} \mathrm{e}(\mathrm{k}-2)+\mathrm{PID}(\mathrm{k}-4)
$$


Donde $k$ representa la muestra presente en un intervalo de $T_{s}$.

La comparación de una plataforma computacional y una plataforma dedicada, es demostrar su desempeño y su propósito para lo que están hechas específicamente, en el cuadro 1 se describen las plataformasde comparación.

Cuadro 1. Características de plataformas en comparación.

\begin{tabular}{|c|c|}
\hline $\begin{array}{l}\text { Windows } 8, \text { NI USB- } 6211,64 \text { bits } \\
\text { Plataforma computacional }\end{array}$ & $\begin{array}{c}\text { FPGA cRIO-9074 } \\
\text { Plataforma FPGA en tiempo real }\end{array}$ \\
\hline $\begin{array}{l}\text { - } \text { Precisión de punto flotante } \\
\text { encodimentación de posición a través de señales de } \\
\text { entradas digitales en la NI USB } 6211 \\
\text { - Salida de valores de control a través de salida de } \\
\text { voltaje analógica } \pm 10 v \text { y } 12 \text { bits para interpretarse } \\
\text { por un circuito electrónico de puente H (L293d) para } \\
\text { controlar el sentido de giro del motor. Tiempo de } \\
\text { muestreo de } 10 \text { ms (mínimo tiempo de muestreo) }\end{array}$ & $\begin{array}{l}\text { - Precisión simple de } 6 \text { cifras significativas } \\
\text { - Realimentación de posición a través de señales de } \\
\text { encoder, procesadas dentro del mismo programa, } \\
\text { - Sontrol en tiempo real y hasta } 400 \mathrm{MHz} \\
\text { tensión analógica } \pm 10 v \text { y } 12 \text { bits para interpretarse } \\
\text { por un circuito electrónico de puente H (L293d) para } \\
\text { controlar el sentido de giro del motor } \\
\text { - Tiempo de muestreo de } 10 \text { ms, } 1 \text { ms y } 100 \text { us } \\
\text { - Plataforma de despliegue robusta y reconfigurable } \\
\text { para aplicaciones embebidas y de control. } \\
\text { - Dispositivos de E/S modular para E/S flexible } \\
\text { - Control en Tiempo Real para implementar } \\
\text { aplicaciones de control de tiempo crítico } \\
\text { - Control dedicado }\end{array}$ \\
\hline
\end{tabular}

El controlador se implementa de forma individual acada actuador de la mMHR. Por la limitante de la plataforma computacional y para establecer una comparación se realizaron pruebas con ciclos a $10 \mathrm{~ms}$. Aunque la ejecución como se ha dicho no está sujeta estrictamente a este tiempo, éste es el que se solicita al programa para ejecutar cada ciclo. 


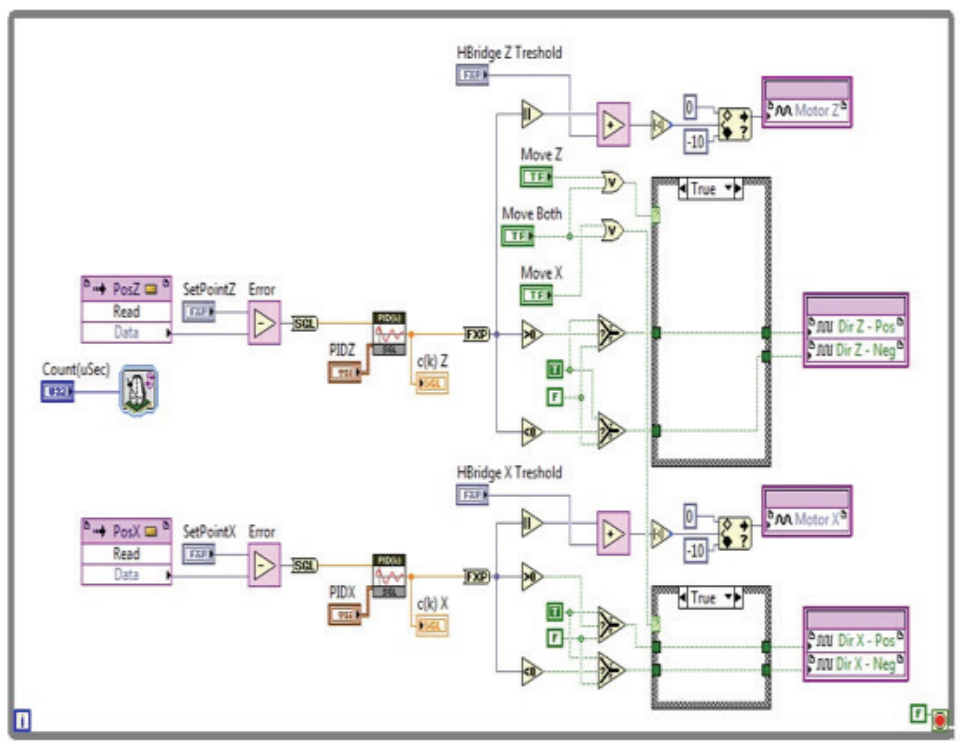

Figura 6. Programa de control en FPGA para la mMHR.

Como de observa en la figura 6, se muestra el programa del control de posición en tiempo real FPGA, que cuenta con las mismas características y parámetros de implementación para la plataforma computacional para evaluar el desempeño de cada plataforma de control.

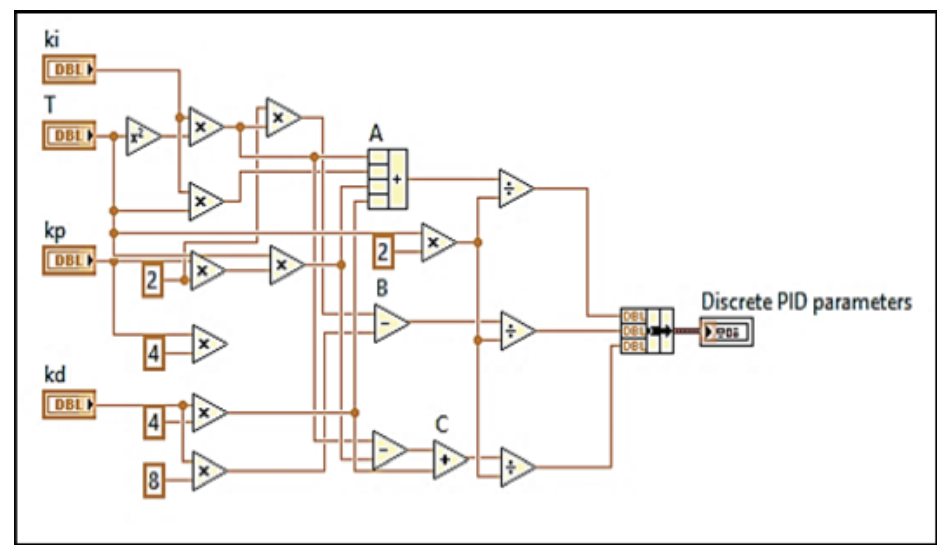

Figura 7. Control PID discreto en FPGA y labVIEW PC.

En la figura 7, se muestra el código del control discreto diseñado, donde se encuentra el control automático de posición de cada eje de movimiento, el control de posición se diseña e implementa de manera independiente a cada eje de movimiento, el entorno ofrece la flexibilidad de modificar los parámetros de control de forma sencilla, con un propósito comparativo dedesempeño. 


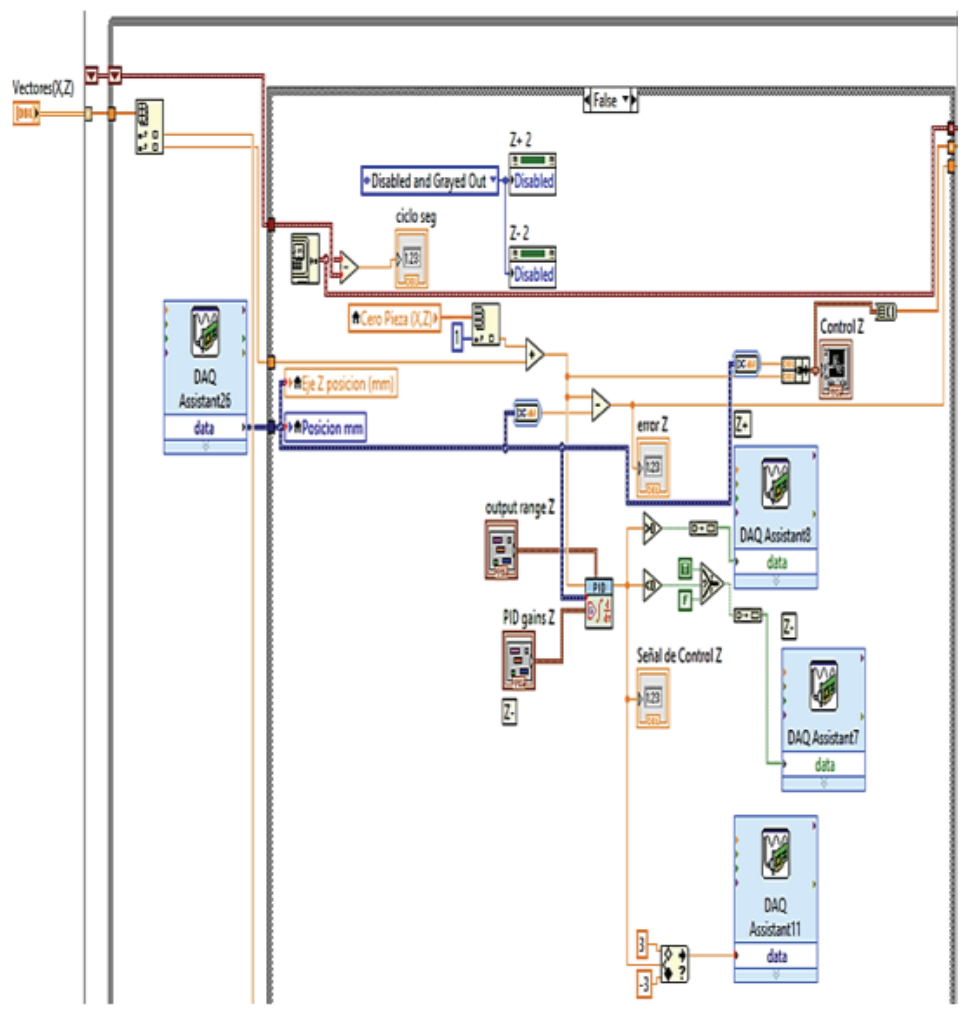

Figura 8. Programa de control en labVIEW en plataforma computacional con DAQ.

En la figura 8, se muestra el programa de control discreto diseñado y que es implementado en plataforma de control computacional con tarjeta de adquisición de datos, bajo las mismas condiciones y parámetros, con el fin de comparar el desempeño del control contra la plataforma de control FPGA en tiempo real y medir los resultados de su respuesta ante un maquinado de una pieza.

En la figura 9 se grafican los resultados que se obtienen al mecanizar una misma pieza prueba, tanto con una plataforma computacional y una plataforma dedicada FPGA los resultados de la pieza medidos con el mismo tiempo de muestreo de $10 \mathrm{~ms}$ son más precisos con la implementación de una plataforma dedicada ya que por las características antes mencionadas es un control dedicado en tiempo real, y que en una plataforma computacional donde el mínimo tiempo de muestreo es de $10 \mathrm{~ms}$ la precisión que se obtuvo es menos porque es una plataforma con un sistema que no garantiza el tiempo de ejecución de sus procesos, los recursos y capacidades son limitados de forma que la precisión de un mecanizado tiene un límite y además no garantiza la misma precisión en cada ejecución. 


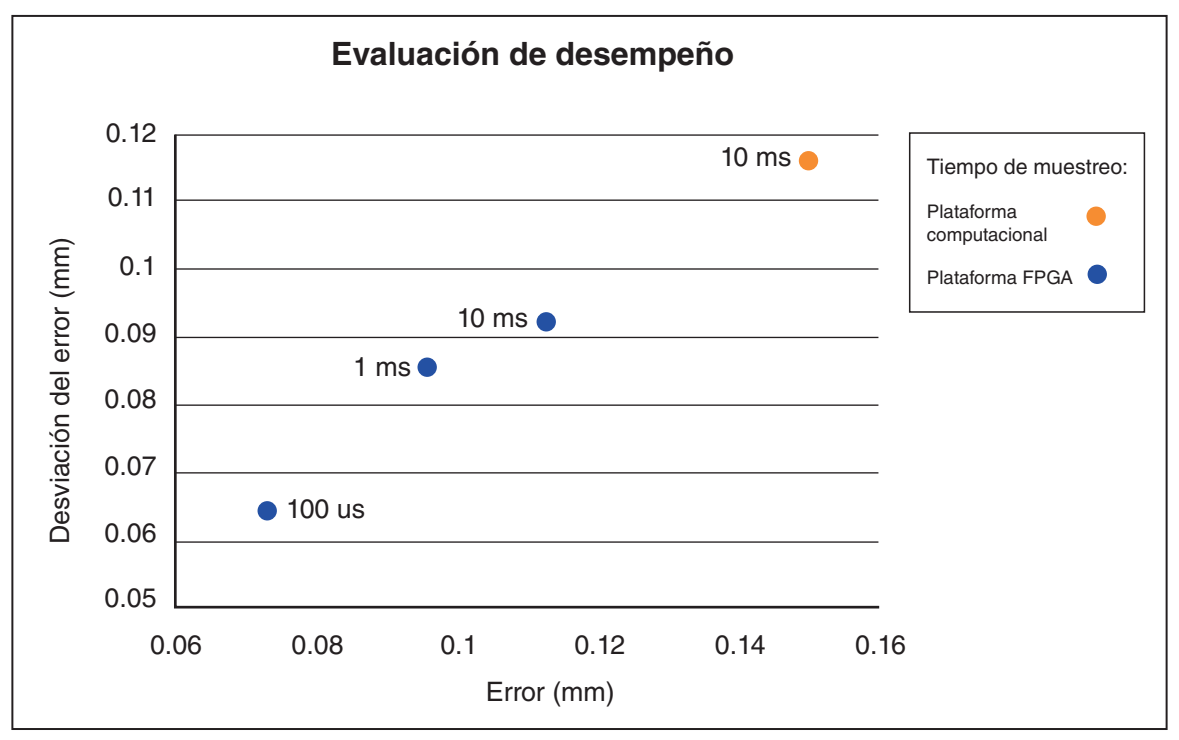

Figura 9. Gráfica de desempeño de control comparativo de plataformas de control.

Por otro lado, se realizaron las mismas pruebas en la plataforma dedicada FPGA con menores tiempos de muestreo y la precisión del mecanizado se vio mejorada. Las pruebas fueron con tiempos de muestreo de $1 \mathrm{~ms}$ y 100 us.

En el desarrollo y evolución de las máquinas-herramienta CNC y micro-máquinas herramientas reconfigurables la creciente tendencia y sobre todo para el beneficio de la industria de manufactura el generar el máximo de producción con los mínimos recursos, esto conlleva en implementar controladores que actúen con la mayor precisión y desempeño en un mínimo de tiempo posible.

Los actuadores son el objetivo principal de aplicación para poder considerar en una máquina su rentabilidad y desempeño energético de costos, así como garantizar una vida útil de diseño. El enfoque propuesto de este trabajo de maquinar una pieza de prueba además de determinar su desempeño y precisión en diferentes plataformas de control es maquinar diferentes materiales y determinar cómo se afectan las variables características del motor y graficar su curva para observar en que zona de puntos de operación trabaja el motor.

\section{Conclusiones}

El control de posición propuesto muestra la ventaja de operar una zona de operación de mayor eficiencia del motor de DC. No solo se obtienen una respuesta de control aceptable de acuerdo con el diseño del controlador, sino que también se logra obtener una operación durante el maquinado de mayor eficiencia. Los sistemas en tiempo real

en FPGA (en este caso MyRio) permiten lograr un maquinado más eficiente y contar con una respuesta con un error de seguimiento menor. La propuesta de incluir en el interpolador las condiciones de zona de operación que limitan los incrementos de los pasos permiten poder garantizar eficiencia de maquinado mayores, así como precisiones en el mismo adecuadas, en el empleo de micro maquinas con máquinas deDC. 


\section{Referencias}

[1] Jang, S., Jung, Y., Hwang, H., y Y. J. Park Choi. Development of a Reconfigurable Micro Machine Tool for Microfactory, Smart Manufacturing Application, ICSMA, pp.190-195. Korea,2008.

[2] Aoyama, H., Fuchiwaki, O., Misaki, D., y Usuda, T. Desktop Micro Machining System by Multiple Micro Robots, IEEE. Japón, 2006.

[3] Suh, M., Kang, S.K., Chung, D.H., Stroud, I.: Theory and Design of CNC Systems. Springer Series in Advanced Manufacturing. Springer, London, UK(2008).

[4] Koren-Yoram, Lo. C. C. Advanced Controller for Feed Drives, CIRP Annals-Manufacturing Technology, Elsevier, 41(2), 689-698, 1992.

[5] Luggen-W. Fundamentals of Numerical Control, N.Y: Editorial Delmar Publishers, 1988-287, ISBN 10:0827331398,1988

[6] Alfonso de Lara R. Diseño e implementación en FPGA del control numérico computarizado para fresadora de tres ejes: control manual e interpretación de coordenadas en dos dimensiones, Universidad Rey Juan Carlos. España,2011.

[7] Hernandez-L.W. Estudio experimental del desgaste del flanco y de las fuerzas de corte en el torneado en seco de alta velocidad del acero AISI 1045, tesis (doctoral), Universidad deHolguin.

[8] Yaodong Tao; Hu Lin; Yi Hu; Xiaohui Zhang; Zhicheng Wang, "Efficient implementation of CNC Position Controller using FPGA," Industrial Informatics, 2008. INDIN 2008. 6th IEEE International Conference on , vol., no., pp.1177,1182,2008

[9] Alfonso de Lara R. Diseño e implementación en FPGA del control numérico computarizado para fresadora de tres ejes: control manual e interpretación de coordenadas en dos dimensiones, Universidad Rey Juan Carlos. España,2011. 